\title{
Prenatal diagnosis of coarctation of the aorta with a long and angled isthmus by two- and three-dimensional echocardiography: a case report
}

\author{
Yu Wang ${ }^{1}$, Caixia Liu', Ying Zhang ${ }^{1 *}$ (I) and Meilian Wang ${ }^{2^{*}}$
}

\begin{abstract}
Background: Prenatal diagnosis of coarctation of the aorta (COA) is challenging for most examiners. The malformation often occurs at the aortic isthmus, which is a short segment between the origin of the left subclavian artery and the insertion of the ductus. We report herein a rare case of CoA with a long, angled, and hypoplastic isthmus. The echocardiographic characteristics and postmortem findings are presented to approach the skill of fetal diagnosis.

Case presentation: A pregnant women undergone fetal echocardiography at $26+3$ gestational weeks in our center. Conventional two-dimensional echocardiography (2DE) showed that ascending aorta went straight upward branching three brachiocephalic arteries without the appearance of the arch, suggesting the possibility of an interrupted aortic arch. Three-dimensional echocardiography (3DE) using spatiotemporal image correlation (STIC) and high-definition flow imaging technique was performed to obtain the 3D rendered images, which clearly showed the arch and its angled junction with the slim isthmus in space. Intra-uterine fetal death occurred and an autopsy was performed. The gross findings showed the angled hypoplastic aortic isthmus in detail and thus confirmed the prenatal diagnosis.

Conclusions: Traditional 2DE may be limited in showing the angled hypoplastic aortic isthmus, while the 3DE STIC technique can provide additional spatial information to show great arteries in detail, help to find tiny vessels, and thus benefit the examiners to make an accurate diagnosis.
\end{abstract}

Keywords: Fetus, Coarctation of the aorta, 3D, Spatiotemporal image correlation

\section{Background}

The aortic isthmus is a part of the aorta locating between the origin of the left subclavian artery and the ductus. In fact, the narrowing occurs in this segment in more than 90\% cases of coarctation of the aorta (CoA) [1]. Usually, the isthmus is very short, only about $1 \mathrm{~cm}$ in length. A discrete stenosis may present when coarctation occurs

\footnotetext{
*Correspondence: baogoubei@hotmail.com; wangmeilian_m@hotmail.com ${ }^{1}$ Department of Ultrasound, Shengjing Hospital of China Medical University, Shenyang, Liaoning, China

${ }^{2}$ Department of Microbiology and Parasitology, College of Basic Medical

Sciences, China Medical University, Shenyang, Liaoning, China
}

at this position. Under very rare conditions, a long, slim and hypoplastic isthmus would appear, forming the junction between the aortic arch and descending aorta. In this study, we report a fetal case of CoA with this "special" isthmus. Conventional two-dimensional echocardiography (2DE) made a diagnosis of interrupted aortic arch (IAA) as the attachment of the arch to the ductus and descending aorta was invisible. Three-dimensional echocardiography (3DE) using spatiotemporal image correlation (STIC) and high-definition flow imaging (HDFI) technology was then performed to obtain the 3D 
reconstructed image, which helped to reach an accurate diagnosis of CoA confirmed by postmortem findings.

\section{Case presentation}

A 32-year-old woman, gravida 3, para 1, was referred to our center at $26+3$ weeks of gestation for further fetal cardiac examination for suspected cardiac anomalies. The patient was in good health without any maternal complications or high-risk factors (e.g. diabetes, hypertension, amniotic disorders). A detailed echocardiogram was performed to find any potential cardiac anomalies using transverse and sagittal scanning. Conventional 2DE was used to show fetal anatomical structures. Color Doppler flow imaging (CDFI), together with HDFI was used to show fetal hemodynamics. The sound beam was continuously moved upward along the vertical axis of the fetal thorax to perform the transverse scanning. The four-chamber view $(4 \mathrm{CV})$ showed a symmetrical left and right heart. The left and right outflow tract views showed normal ventriculoarterial connections. However, a small-sized ventricular septal defect (VSD) was identified at the $4 \mathrm{CV}$ and the left outflow tract view with the communication clearly shown by CDFI (Fig. 1). An additional movie file shows this in more detail (See Additional file 1: Video). The pulmonary valve annulus (PVA) was apparently wider in comparison with the aortic valve annulus (AVA). The ratio of PVA/AVA was 1.6 in diameter. The size of the pulmonary artery (PA) was also larger than the aorta (AO) and the ratio of $\mathrm{PA} / \mathrm{AO}$ was 2.1 in diameter. At the three-vessel trachea (3VT) view, the widened pulmonary trunk continued to be the ductus, which was connected with the descending aorta. At the same time, a tiny vessel was found located at the right side of the pulmonary trunk, with no convergence with the ductus (Fig. 2). An additional movie file shows this in more detail (See Additional file 2: Video). Under normal conditions, the aortic arch joins with the ductus to form proximal descending aorta in a characteristic "V" shape at the 3VT view [2]. The echocardiographic manifestations in the current case thus suggest the possibility of an IAA. Sagittal planes were also scanned to obtain more information. The arch did not present, instead, the ascending aorta was visualized going straight upward and then branching three brachiocephalic arteries in a "W" shape (Fig. 3). An additional

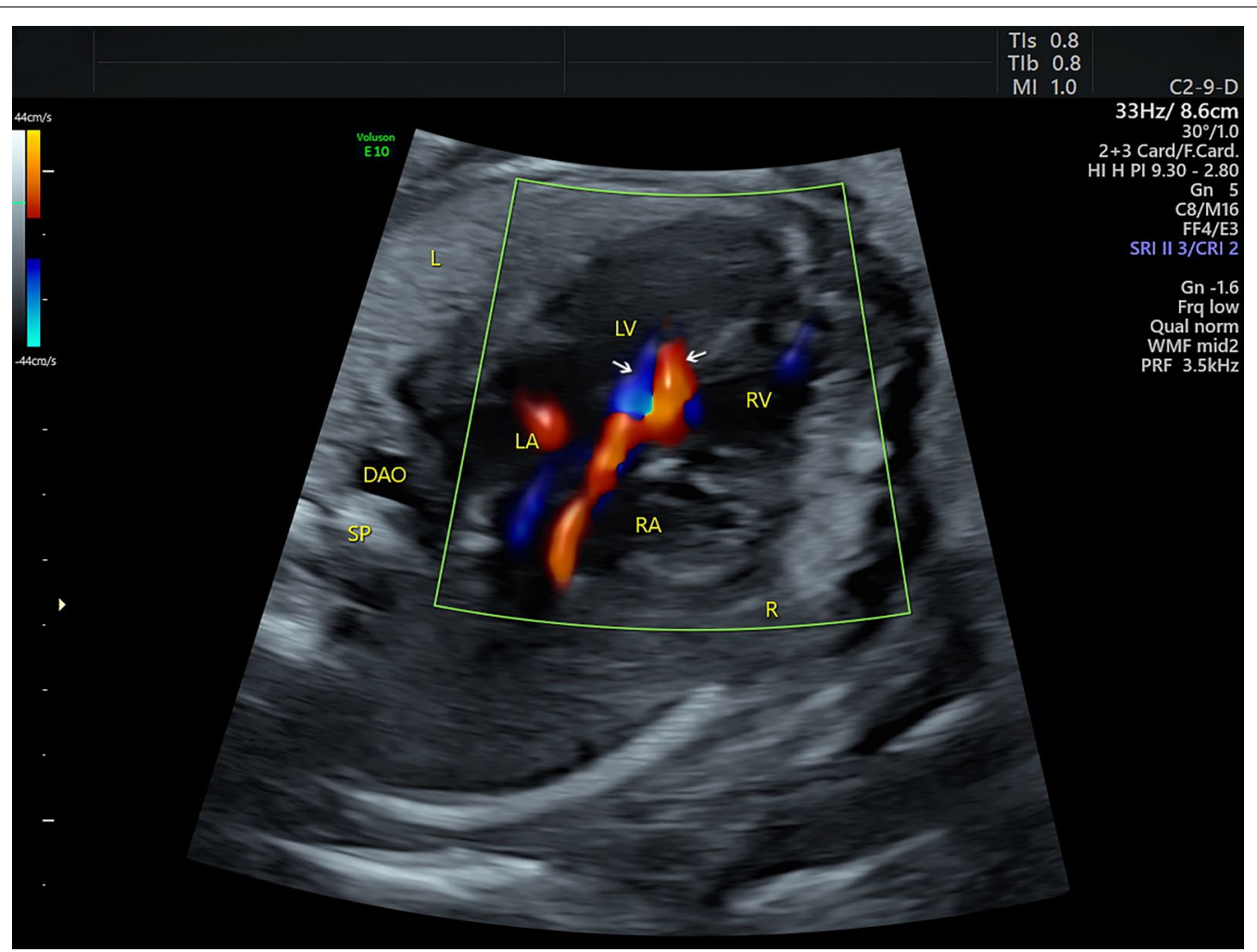

Fig. 1 Sonograms of the fetus of coarctation of the aorta at the four-chamber view. The four-chamber view shows symmetrical left and right chambers. In addition, a bidirectional shunt (indicated by the arrows) between the left and right ventricles is demonstrated by color Doppler. DA: ductus arteriosus; DAO: descending aorta; L: left; LA: left atrium; LV: left ventricle; R: right; RA: right atrium; RV: right ventricle; SP: spine 


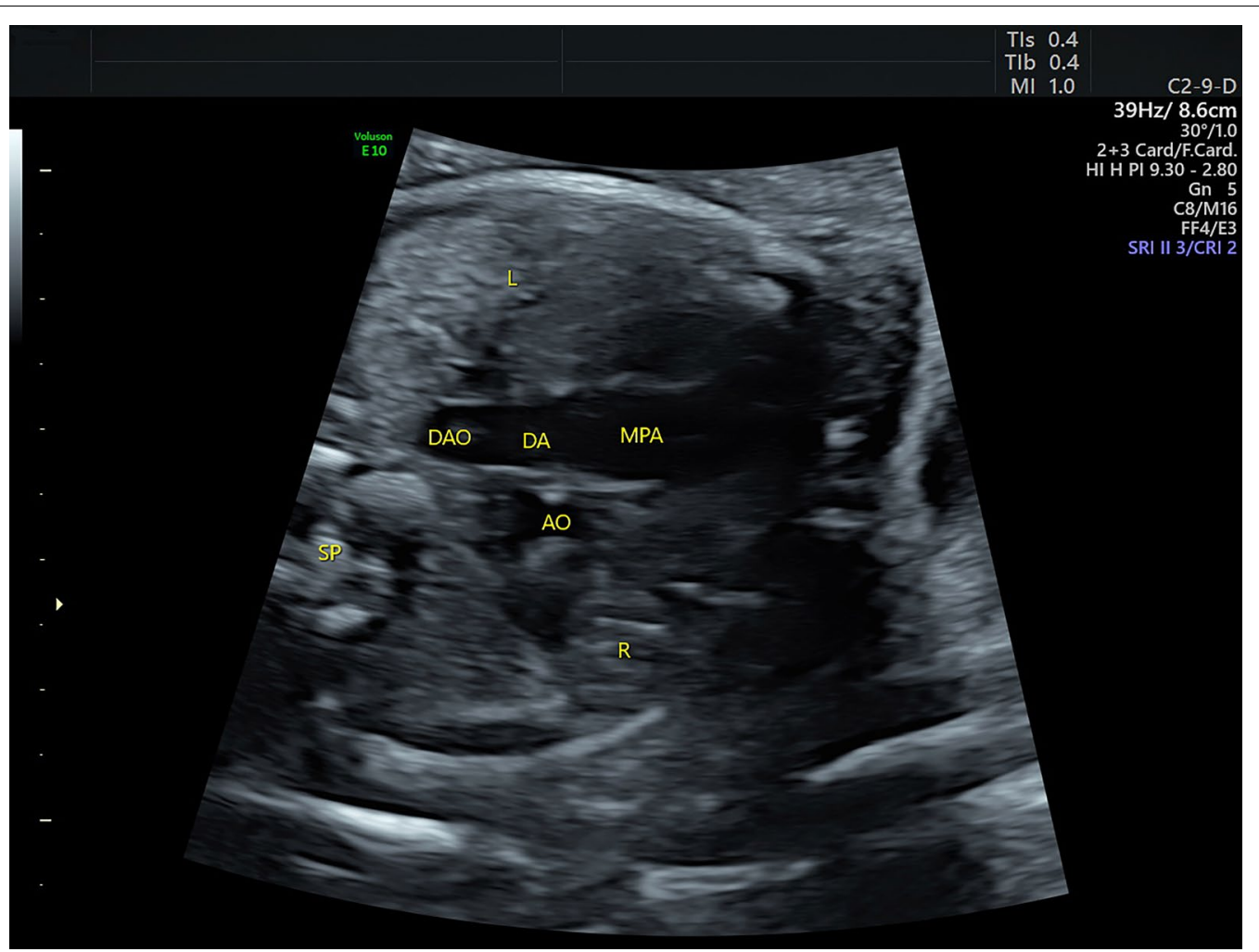

Fig. 2 Sonograms of the fetus of coarctation of the aorta at the three-vessel trachea view. The large-sized pulmonary artery is visualized to connect with the descending aorta via the ductus. The aorta is on the right side of the pulmonary artery but is not visualized to drain into the ductus. AO: aorta; DAO: descending aorta; L: left; MPA: main pulmonary artery; R: right; SP: spine

movie file shows this in more detail (See Additional file 3: Video). A preliminary diagnosis of IAA type A was then reached. The 3DE with STIC technology was then used to obtain 3D images of the great arteries to confirm the 2D diagnosis. A 3D motorized transducer $(4-8 \mathrm{MHz})$ was used to acquire cardiac volumes when scanning the sagittal planes using HDFI. The acquisition time was set to $12.5 \mathrm{~s}$ and the sweep angle was set to $30^{\circ}$. Cardiac volumes were acquired automatically and then reconstructed to display in a cine loop in multiplanar mode. Volume post-analysis was then performed using an off-line software (4D viewer, version 14.0) to obtain the $3 \mathrm{D}$ reconstructed images. This could be achieved by properly adjusting the direction and size of the region of interest (ROI) and the rotation of the images in three orthogonal planes in the volume. A combination of smooth surface and gradient light algorithms was also used to enhance the 3D effect of the reconstructed images. The 3D image demonstrated an abnormal angle of attachment of the aortic arch to the ductus and descending aorta via a slim isthmus (Fig. 4). An additional movie file shows this in more detail (See
Additional file 4: Video). The final diagnosis was CoA at the isthmus.

The patient refused a chromosomal examination and did not go back to the following-up echo four weeks later for personal reasons. She came to the hospital at 31 gestational weeks as she could not feel fetal movement. Intra-uterine fetal death was demonstrated by fetal echo and proofs of intra-uterine infection were found during the induction of the labor. An autopsy was then performed and showed the great arteries in detail. The spatial relationships of the aorta and the pulmonary artery were normal. The size of the ascending aorta was approximately half of that of the pulmonary trunk while it went straight upward and gave off the innominate artery, left common carotid artery and the left subclavian artery, in turn. An abnormal angle of the connection between the aortic arch and the slim isthmus could be appreciated (Fig. 5). In addition, a perimembranous VSD about $4 \mathrm{~mm}$ in diameter was also found. The gross findings confirmed the prenatal diagnosis. 


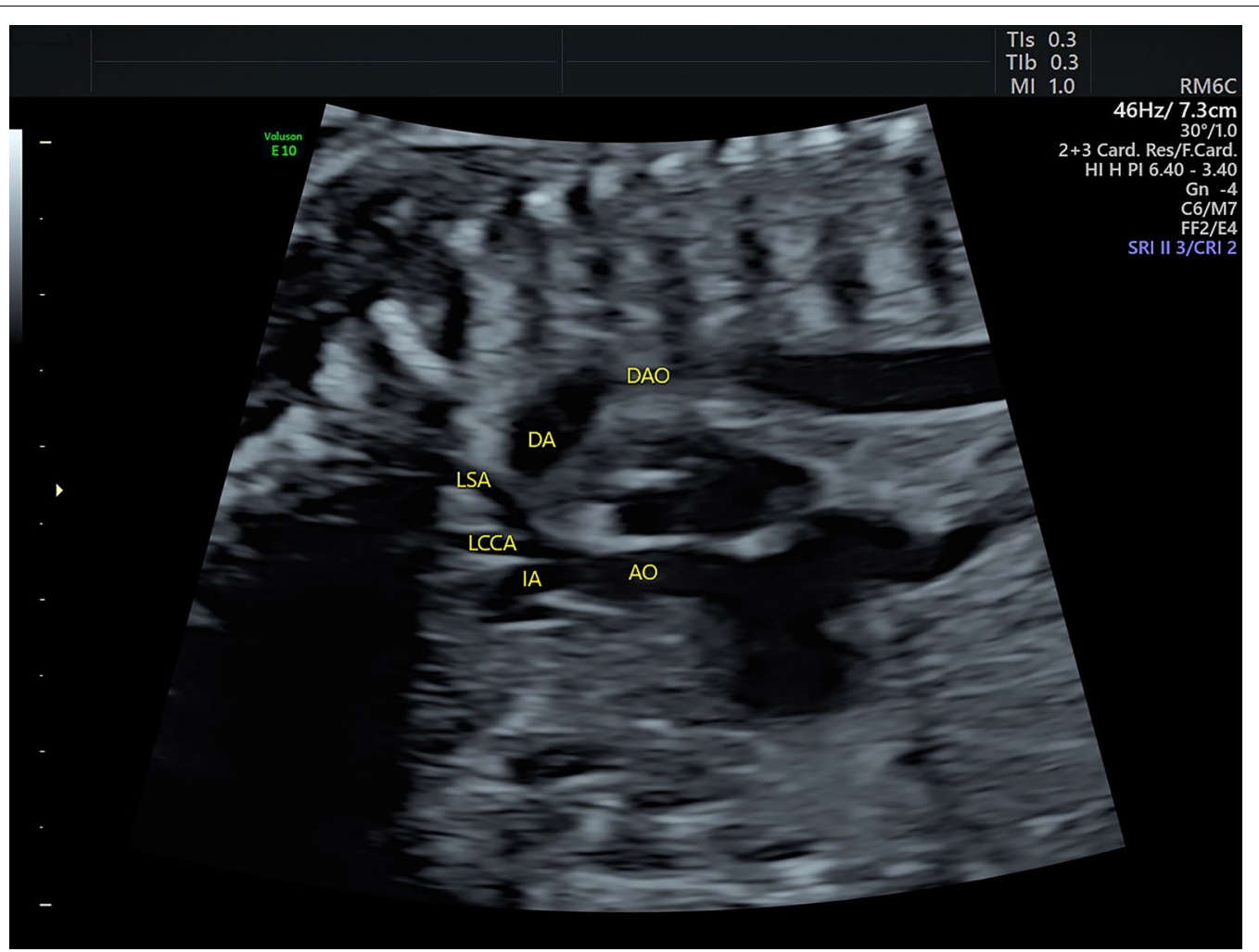

Fig. 3 Sonograms of the fetus of coarctation of the aorta at the sagittal view. The sagittal view shows that the ascending aorta goes straight upward and gives off three brachiocephalic arteries while the aortic arch does not appear. AO: aorta; DA: ductus arteriosus; DAO: descending aorta; IA: innominate artery; LCCA: left common carotid artery; LSA: left subclavian artery

\section{Discussion}

CoA is a form of deformity that stenosis presents in a segment of the aortic lumen along the arch, which may lead to an obstruction of the blood flow. It occurs in about $6-8 \%$ of live births with congenital heart disease [1]. In general, CoA can be classified into three types according to the location of the narrowing relative to the ductus. The first one is the pre-ductal coarctation in which the narrowing presents proximal to the insertion of the ductus. The second form is the ductal coarctation where the narrowing occurs at the junction of the ductus. This type of coarctation is usually associated with the prominent posterior infolding of the aortic wall (the posterior shell). The last type is the post-ductal coarctation, in which the narrowing occurs distal to the insertion of the ductus. For the three types of CoA, the pre-ductal coarctation is most common in utero.

Fetal diagnosis of CoA is generally inferred from secondary findings of ventricular and great artery disproportion [3-6]. Right-sided structures dilatation relative to the left-sided structures usually serves as the first clue to the diagnosis. Previous studies indicated a larger ratio of right ventricle/left ventricle (>1.5) [4] and PVA/AVA
(>1.6) [5-7] in diameter for CoA fetuses, which could be assessed at the $4 \mathrm{CV}$ and the outflow tracts views, respectively. Note that the size discrepancy may not be obvious for bilateral ventricles in the presence of a VSD and the first sign is the great artery disproportion. Our result is consistent with this point. Aortic isthmus diameter $\mathrm{Z}$ scores could be measured either in the sagittal view or the $3 \mathrm{VT}$ view, which are significantly lower in fetuses with CoA $(<3$ standard deviations than normal fetuses with the same gestational age) [7, 8]. A larger ratio $(>1.4)$ of ductus/aortic isthmus in diameter could also suggest the presence of CoA [4]. In addition, the larger distance between the left common carotid artery and the left subclavian artery [8] and some intracardiac complications (e.g. VSD, persistent left superior vena cava, and bicuspid aortic valve) [3] should also call attention to the possibility of CoA. Though we discuss above the needed echo parameters, capabilities, and some criteria to evaluate fetal CoA, it is really not easy to make an accurate prediction or diagnosis in utero. Wide differential diagnosis including pulmonary dilatation (e.g. pulmonary hypertension, premature ductal constriction) is required [9]. Fetal blood redistribution may also contribute to the 


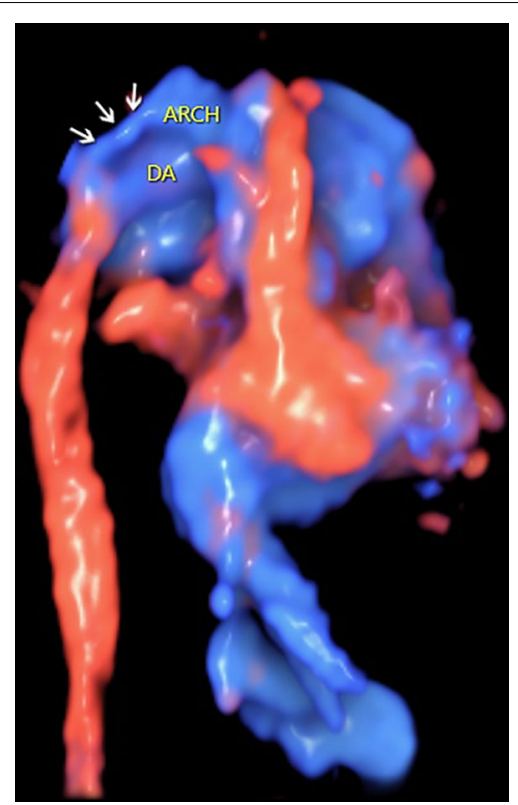

Fig. 4 The 3D reconstructed image showing the great arteries of the fetus with coarctation of the aorta. The 3D rendered image clearly shows the great arteries in space. In addition, it demonstrates a sharp angle between the aortic arch and the slim isthmus (indicated by the arrows). Arch: aortic arch; DA: ductus arteriosus

false diagnosis of fetal CoA. Compression of fetal neck by the umbilical cord might result in a disproportion of cardiac flow, "mimicking CoA", as reported by Więckowska et al. [10]. In addition, different hemodynamics patterns may present for fetuses and neonates when the ductus closes. It is better to be considered as a provisional diagnosis for CoA in utero, which should be either confirmed or refuted after birth as a high false-positive diagnosis is common in fetal life.

We want to stress that CoA is difficult to differentiate from IAA type A in some cases, as the site of the lesion is at the isthmus for both circumstances. For embryonic development, different degrees of regression of the left dorsal aorta at this specific segment lead to the formation of a narrowing aortic lumen, an atretic fibrous band or complete absence of that portion of the arch [11]. Slodki et al. [12] suggested that a large discrepancy between PA/ AO diameters should raise the suspicion of aortic arch anomalies. In their studies, the calculated ratio of PA/ AO was about 2.1 in IAA type A fetuses. Similar disproportional great arteries were found in the current COA fetus, which suggested an insufficient proof to make a differential diagnosis based only on scanning the threevessel view. It is necessary to move the sound beam more cephalad to determine whether the ductus join with aorta at the $3 \mathrm{VT}$ view. The disjoint of the two great arteries suggests the possibility of an inexistent arch. However, it might also be possible that the arch and/or the isthmus is too slim to be seen in this plane in some rare conditions. Sagittal planes may be useful in showing the course and branching of the aorta. Previous report [13] indicated that the ascending aorta goes straight upward to the fetal
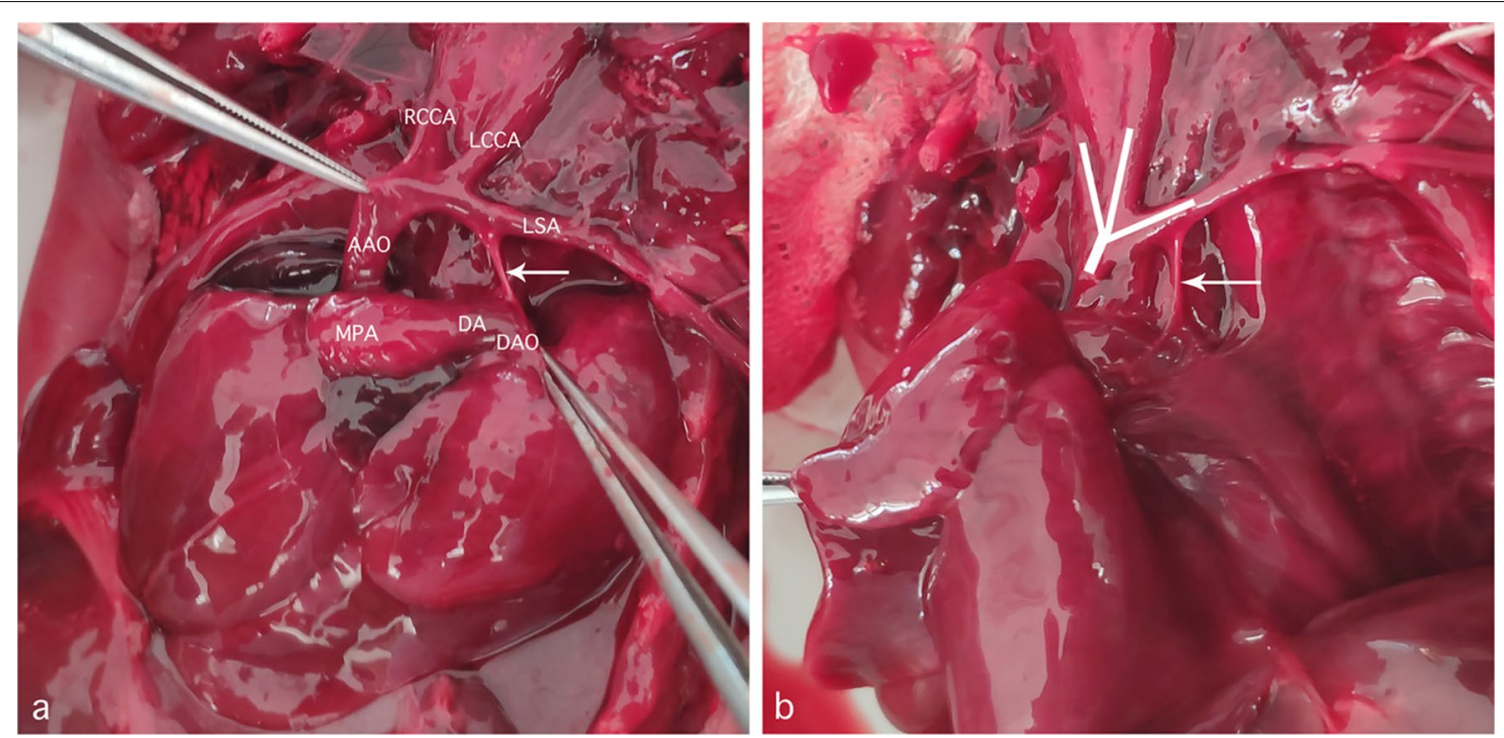

Fig. 5 Gross findings of the fetus of coarctation of the aorta. The branching patterns of aortic arch and the slim isthmus (indicated by the arrow), together with its connection with descending aorta are clearly shown, when pulling up the great arteries using two forceps (a). The original position of the great arteries is also shown (b). Three white lines are drawn to show the aortic arch branches, which are presenting in a "W" shape. The course of the arch is not transverse but oblique upward, leading to a very sharp angle of its connection with the isthmus (indicated by the arrow) 
head without the appearance of a curved arch in terms of IAA. However, we found that the course and angle of ascending aorta in current CoA fetus seem like those of IAA, which adds the difficulty to make a differentiation. In fact, the gross finding shows a long isthmus with a relatively short arch. The course of the arch is not transverse but oblique upward, leading to a very sharp angle of its connection with the isthmus, in comparison with the normal smooth junction between the adjacent structures. Based on these points, the arch was misrecognized as parts of the ascending aorta with the angled slim isthmus invisible during the sagittal scanning.

Volume sonography is the 3D technique used in fetal echocardiography [14]. It was initially proposed as a concept of research but now have been proved to provide much diagnostic information in addition to conventional 2DE. Briefly, STIC technology is used to acquire datasets containing many $2 \mathrm{D}$ images and then combine into a single cardiac volume $[15,16]$. It has also been adapted to acquire volumes with color information, including color Doppler, HDFI, and B-flow imaging. In our previous studies, we have shown that the 3D technique helped to diagnose fetal tubular arch hypoplasia [17], IAA [13], and arch anomalies [18]. Herein, we further proved its value in the diagnosis of fetal CoA. The narrowing aortic lumen was clearly shown with blood filling, indicating a stenosis isthmus instead of an interruption. The anatomical structures and spatial relationships of the great arteries could be vividly shown in rendered HDFI images in an in-depth perspective, which resembles the autopsy findings very much. Another benefit, the addition of depth perception in the 3D reconstructed image actually shows anatomical information of multiple 2D planes, some of which could not be obtained during the routine $2 \mathrm{D}$ scan. We speculate it is the reason for the correct diagnosis of the $3 \mathrm{D}$ modality in the current report.

Previous reports had indicated that prenatal diagnosis of CoA improves survival and reduces morbidity $[19,20]$. We report herein an accurate diagnosis of this malformation by 3D modality, which also ensured a better prenatal counseling for the parents, it is a benefit of our research. In addition, it is extremely rare that intrauterine death occurred for CoA fetuses, which is not reported before. We expect this "special" case of fetal CoA may benefit screening sonographers and obstetricians.

\section{Conclusion}

We present a fetal case of CoA diagnosed by 2DE and 3DE-STIC technology. The traditional 2DE has some limitations in showing the angled and hypoplastic aortic isthmus, while the $3 \mathrm{D}$ rendered image may provide additional spatial information to show great arteries in detail, help to find tiny vessels, and thus benefit the examiners to make an accurate diagnosis.

\section{Abbreviations}

2DE: Two-dimensional echocardiography; 3DE: Three-dimensional echocardiography; 3VT: Three-vessel trachea ; 4CV: Four-chamber view; AO: Aorta; CDFI: Color Doppler flow imaging; CoA: Coarctation of the aorta; HDFI: High-definition flow imaging; IAA: Interrupted aortic arch; PA: Pulmonary artery; STIC: Spatiotemporal image correlation; VSD: Ventricular septal defect.

\section{Supplementary Information}

The online version contains supplementary material available at https://doi. org/10.1186/s12872-021-01987-7.

Additional file 1. Video. Sonograms showing symmetrical left and right ventricles and bidirectional communication between the two ventricles at the four-chamber view.

Additional file 2. Video. Sonograms showing the three-vessel trachea view in a fetus with coarctation of the aorta. The large-sized pulmonary artery is visualized to connect the descending aorta via the ductus. The aorta is on the right side of the pulmonary artery but is not visualized to drain into the ductus.

Additional file 3. Video. Sonograms showing the sagittal view in a fetus with coarctation of the aorta. It shows that the ascending aorta goes straight upward and gives off three brachiocephalic arteries while the aortic arch does not appear.

Additional file 4. Video. The 3D rendered image cine loops showing the great arteries in a fetus with coarctation of the aorta. The 3D rendered image clearly shows the great arteries in space. In addition, it demonstrates a sharp angle between the aortic arch and the slim isthmus (indicated by the arrows). Arch: aortic arch; DA: ductus arteriosus.

\section{Acknowledgements}

We wish to acknowledge Letpub for its linguistic assistance during the preparation of this manuscript.

\section{Authors' contributions}

$Y Z, Y W$ and $C X L$ drafted the manuscript. YZ performed the fetal echocardiography. YW performed the 3D post-analysis of the cardiac volumes. MLW performed the autopsy work. All authors read and approved the final manuscript.

\section{Funding}

YW was funded by 345 Talent Project and Science Foundation for The Excellent Youth Scholars of China Medical University (No. QGZD2018065). YZ was supported by grants from scientific research projects sponsored by the Science and Technology Agency of Liaoning province (No. 2012225098), and sponsored by the Science and Technology Agency of Shenyang, China (No. F16-206-9-11). The funders had no role in study design, data collection and analysis, decision to publish, or preparation of the manuscript.

\section{Availability of data and materials}

The datasets supporting the conclusions of this article are included within the manuscript (and its additional files). The authors would like to share raw anonymized video data related to the current study, which could only be used for personal study. The demanders may contact baogoubei@hotmail.com.

\section{Declarations}

\section{Ethics approval and consent to participate}

This study was approved by the Ethics Committee of Shengjing Hospital of China Medical University. Written informed consent was obtained from the parents for publication of clinical details, clinical images, and videos. 


\section{Consent for publication}

As the patient was a fetus, informed written consent was obtained from the mother (legal guardian) for publication of this report and any accompanying clinical data, images and videos.

\section{Competing interests}

The Authors declare that there is no competing interest.

Received: 24 July 2020 Accepted: 7 April 2021

Published online: 13 April 2021

\section{References}

1. Beekman $\mathrm{RH}$, et al. Coarctation of the aorta. In: Allen HD, Driscoll D, Shaddy RE, et al., editors. Moss and Adams's heart disease in infants, children and adolescents: including the fetus and young adult, vol. 2. 8th ed. Philadelphia: Lippincott Williams \& Wilkins; 2013.

2. Yoo SJ, Min JY, Lee YH, Roman K, Jaeggi E, Smallhorn J. Fetal sonographic diagnosis of aortic arch anomalies. Ultrasound Obstet Gynecol. 2003;22(5):535-46.

3. Familiari $\mathrm{A}$, Morlando $\mathrm{M}$, Khalil $\mathrm{A}$, et al. Risk factors for coarctation of the aorta on prenatal ultrasound: a systematic review and meta-analysis. Circulation. 2017:135(8):772-85.

4. Mărginean C, Mărginean CO, Muntean I, Togănel R, Voidăzan S, Gozar L. The role of ventricular disproportion, aortic, and ductal isthmus ultrasound measurements for the diagnosis of fetal aortic coarctation, in the third trimester of pregnancy. Med Ultrason. 2015;17(4):475-81.

5. Durand I, Deverriere G, Thill C, Lety AS, Parrod C, David N, Barre E, Hazelzet T. Prenatal detection of coarctation of the aorta in a non-selected population: a prospective analysis of 10 years of experience. Pediatr Cardiol. 2015;36(6):1248-54

6. Slodki M, Rychik J, Moszura T, Janiak K, Respondek-Liberska M. Measurement of the great vessels in the mediastinum could help distinguish true from false-positive coarctation of the aorta in the third trimester. J Ultrasound Med. 2009;28(10):1313-7.

7. Gómez-Montes E, Herraiz I, Gómez-Arriaga PI, Escribano D, Mendoza A, Galindo A. Gestational age-specific scoring systems for the prediction of coarctation of the aorta. Prenat Diagn. 2014;34(12):1198-206.

8. Sivanandam S, Nyholm J, Wey A, Bass JL. Right ventricular enlargement in utero: is it coarctation? Pediatr Cardiol. 2015;36(7):1376-81.

9. Swięchowicz B, Respondek-Liberska M. Fetal "aortic coarctation" and different neonatal follow-up in 3 cases. Prenat Cardio. 2017;7(1):65-72.

10. Więckowska K, Zych-Krekora K, Słodki M, Respondek-Liberska M. Do umbilical cord wrapped around the fetal body can mimic signs of aortal coarctation? Prenat Cardio. 2016:6(1):82-6.
11. Weinberg PM. Aortic arch anomalies. In: Allen HD, Driscoll DJ, Shaddy RE, Feltes TF, editors. Moss and Adams' heart disease in infants, children, and adolescents: including the fetus and young adults. Philadelphia: Lippincott Williams \& Wilkins; 2008. p. 731-60.

12. Slodki M, Moszura T, Janiak K, Sysa A, Seligman NS, Weiner S, RespondekLiberska M. The three-vessel view in the fetal mediastinum in the diagnosis of interrupted aortic arch. Ultrasound Med Biol. 2011;37(11):1808-13.

13. Zhang D, Zhang Y, Ren W, Sun F, Guo Y, Sun W, Wang Y, Huang L, Cai A. Prenatal diagnosis of fetal interrupted aortic arch type A by two-dimensional echocardiography and four-dimensional echocardiography with B-flow imaging and spatiotemporal image correlation. Echocardiography. 2016;33(1):90-8.

14. Adriaanse BM, van Vugt JM, Haak MC. Three- and four-dimensional ultrasound in fetal echocardiography: an up-to-date overview. J Perinatol. 2016;36(9):685-93.

15. Ahmed $\mathrm{BI}$. The new 3D/4D based spatio-temporal imaging correlation (STIC) in fetal echocardiography: a promising tool for the future. J Matern Fetal Neonatal Med. 2014;27(11):1163-8.

16. Araujo Júnior E, Tonni G, Bravo-Valenzuela NJ, Da Silva CF, Meagher $\mathrm{S}$. Assessment of fetal congenital heart diseases by 4-dimensional ultrasound using spatiotemporal image correlation: pictorial review. Ultrasound Q. 2018;34(1):11-7.

17. Zhang Y, Cai A, Wu H, Sun W, Guo Y. Prenatal diagnosis of a fetal tubular arch hypoplasia with an aberrant right subclavian artery: two " $\vee$ " shape structures arising from the transverse arch. Prenat Diagn. 2011;31(6):595-7.

18. Wang Y, Fan M, Siddiqui FA, Wang M, Sun W, Sun X, Lei W, Zhang Y. Strategies for accurate diagnosis of fetal aortic arch anomalies: benefits of three-dimensional sonography with spatiotemporal image correlation and a novel algorithm for volume analysis. J Am Soc Echocardiogr. 2018;31(11):1238-51.

19. Franklin O, Burch M, Manning N, Sleeman K, Gould S, Archer N. Prenatal diagnosis of coarctation of the aorta improves survival and reduces morbidity. Heart. 2002;87(1):67-9.

20. Słodki M, Rizzo G, Augustyniak A, Seligman NS, Zych-Krekora K, Respondek-Liberska M; International Prenatal Cardiology Collaboration Group. Retrospective cohort study of prenatally and postnatally diagnosed coarctation of the aorta (COA): prenatal diagnosis improve neonatal outcome in severe CoA. J Matern Fetal Neonatal Med. 2020;33(6):947-951.

\section{Publisher's Note}

Springer Nature remains neutral with regard to jurisdictional claims in published maps and institutional affiliations.
Ready to submit your research? Choose BMC and benefit from:

- fast, convenient online submission

- thorough peer review by experienced researchers in your field

- rapid publication on acceptance

- support for research data, including large and complex data types

- gold Open Access which fosters wider collaboration and increased citations

- maximum visibility for your research: over 100M website views per year

At $B M C$, research is always in progress.

Learn more biomedcentral.com/submissions 